\title{
Design of triple-band CMOS GPS receiver RF front-end
}

\author{
Ikkyun Jo $^{1 a)}$, Jungnam Bae ${ }^{1}$, Toshimasa Matsuoka ${ }^{1}$, \\ and Takuji Ebinuma ${ }^{2}$ \\ ${ }^{1}$ Department of Electrical, Electronic and Information Engineering \\ Graduate School of Engineering, Osaka University, \\ 2-1 Yamada-oka, Suita, Osaka 565-0871, Japan \\ ${ }^{2}$ Department of Aeronautics and Astronautics \\ Graduate School of Engineering, The University of Tokyo, \\ 7-3-1 Hongo, Bunkyo-ku, Tokyo 113-8656, Japan \\ a) jo@si.eei.eng.osaka-u.ac.jp
}

Abstract: This letter describes the design of a triple-band Global Positioning System (GPS) receiver that simultaneously covers the L1, L2 and L5 frequency bands. The proposed receiver uses an imagerejection technique that can separate signals from the three frequency bands to three corresponding ports. It uses a single RF path containing a low-noise amplifier (LNA), and active and passive mixers with a pair of local oscillator signals. The triple-band GPS RF front-end chip is fabricated using $130 \mathrm{~nm}$ CMOS technology, and has a noise figure of less than $7.1 \mathrm{~dB}$ and an $S_{11}$ coefficient of less than $-10 \mathrm{~dB}$ in the frequency range 1.15-1.6 GHz. The experimental results demonstrate a $35-40 \mathrm{~dB}$ image rejection ratio at each output port with a power consumption of $7.2 \mathrm{~mW}$ (LNA and mixers) using a $1.2 \mathrm{~V}$ supply voltage.

Keywords: GPS, triple-band receiver, RF front-end, CMOS integrated circuit

Classification: Microwave and millimeter wave devices, circuits, and systems

\section{References}

[1] T. Kadoyama, N. Suzuki, N. Sasho, H. Iizuka, I. Nagase, H. Usukubo, and M. Katakura, "A complete single-chip GPS receiver with 1.6-V 24$\mathrm{mW}$ radio in 0.18- $\mu \mathrm{m}$ CMOS," IEEE J. Solid-State Circuits, vol. 39, no. 4, pp. 562-568, April 2004.

[2] T. Ebinuma and A. Yasuda, "Airborne GPS Reflectometry from Low Altitude Aircraft," SICE J. Control, Measurement, and System Integration, vol. 3, no. 6, pp. 429-434, Nov. 2010.

[3] S. Gunawardena, Z. Zhu, and M. Braasch, "Observing the GPS L5 Test Transmission from SVN49 Using Software Radio Processing," InsideGNSS, vol. 4, no. 3, pp. 22-29, May/June 2009.

[4] J. Ko, J. Kim, S. Cho, and K. Lee, "A $19 \mathrm{~mW} 2.6 \mathrm{~mm}^{2}$ L1/L2 Dual Band CMOS GPS Receiver," IEEE J. Solid-State Circuits, vol. 40, no. 7, pp. 1414-1424, July 2005. 
[5] Y. Utsurogi, M. Haruoka, T. Matsuoka, and K. Taniguchi, "CMOS Front-end Circuits of Dual-band GPS Receiver," IEICE Trans. Electron., vol. E88-C, no. 6, pp. 1275-1279, June 2005.

[6] M. Detratti, E. López, E. Pérez, and R. Palacio, "Dual-Band RF FrontEnd Solution for Hybrid Galileo/GPS Mass Market Receivers," 5th IEEE Consumer Communications and Networking Conference (CCNC 2008), pp. 603-607, Jan. 2008.

[7] J. Wu, P. Jiang, D. Chen, and J. Zhou, "A Dual-Band GNSS RF Front-End With a Pseudo-Differential LNA," IEEE Trans. Circuits Syst., vol. 58, no. 3, pp. 134-138, March 2011.

[8] D. Chen, W. Pan, P. Jiang, J. Jin, T. Mo, and J. Zhou, "Reconfigurable Dual-Channel Multiband RF Receiver for GPS/Galileo/BD-2 Systems," IEEE Trans. Microw. Theory Tech., vol. 60, no. 11, pp. 3491-3501, Nov. 2012.

[9] T. Elesseily, T. Ali, and K. Sharaf, "A crystal-tolerant fully integrated CMOS low-IF dual-band GPS receiver," Analog Integrated Circuits and Signal Processing, vol. 63, no. 2, pp. 143-159, May 2010.

[10] M. Haruoka, Y. Utsurogi, T. Matsuoka, and K. Taniguchi, "A Study on the LO Phase Error Compensation of GPS Dual-Band Image-Reject Mixer," IEICE Trans. Electron. (Japanese Edition), vol. J86-C, no. 11, pp. 1177-1183, Nov. 2003.

[11] M. Haruoka, Y. Utsurogi, T. Matsuoka, and K. Taniguchi, "A Study on the LO Phase Error Compensation of GPS Dual-Band Image-Reject Mixer," Electronics and Communications in Japan, Part 2, vol. 88, no. 12 , pp. 26-33, Dec. 2005.

[12] K. W. Martin, "Complex Signal Processing is Not Complex," IEEE Trans. Circuits Syst. I, vol. 51, no. 9, pp. 1823-1836, Sept. 2004.

[13] B. Boashash, "Estimating and Interpreting The Instantaneous Frequency of a Signal-Part 1: Fundamentals," Proc. IEEE, vol. 80, no. 4, pp. 520538, April 1992.

[14] D. K. Shaeffer and T. H. Lee, "A 1.5-V, 1.5-GHz CMOS Low Noise Amplifier," IEEE J. Solid-State Circuits, vol. 32, no. 5, pp. 745-759, May 1997.

[15] D. K. Shaeffer and T. H. Lee, "Corrections to "A 1.5-V, 1.5-GHz CMOS Low Noise Amplifier"," IEEE J. Solid-State Circuits, vol. 40, no. 6, pp. 1397-1398, May 2005.

[16] T. Kamata, K. Okui, M. Fukasawa, T. Matsuoka, and K. Taniguchi, "Low-Power Zero-IF Full-segment ISDB-T CMOS Tuner with Tenthorder Baseband Filters," IEEE Trans. Consum. Electron., vol. 57, no. 2, pp. 403-410, May 2011.

[17] G. Kim, B. Murakami, M. Goto, T. Kihara, K. Nakamura, Y. Shimizu, T. Matsuoka, and K. Taniguchi, "Small-Signal and Noise Model of FDSOI MOS Devices for Low Noise Amplifier," Jpn. J. Appl. Phys., PT.1, vol. 45, no. 9A, pp. 6872-6877, Sept. 2006.

[18] B. Wei, Y. Dai, J. Wang, T. Matsuoka, and K. Taniguchi, "Design of a low-voltage CMOS mixer based on variable load technique," IEICE Electron. Express, vol. 7, no. 7, pp. 473-479, April 2010.

\section{Introduction}

Recently, the Global Positioning System (GPS), which was originally developed for military purposes, is widely used for obtaining location information 
in applications such as car navigation systems. Consumer applications generally employ a single GPS receiver chip fabricated using a CMOS process. The absence of compound semiconductors or bipolar processes leads to a significant reduction in the size, cost, and power consumption of such devices [1], allowing the widespread use of civilian GPS signals.

On the other hand, the use of civilian GPS signals is also becoming attractive for scientific applications, such as ocean remote sensing [2], for which higher positioning accuracy is required. One way of achieving this is to use multiple civilian GPS signals at different frequencies, and such an approach can also offer advantages for robust GPS services such as those used in aviation. Multi-band GPS has come closer to reality with the launch of a satellite transmitting in the L5 band, to compliment the L1 and L2 civilian bands that are already in use [3]. Thus, GPS receivers that can detect all three bands simultaneously are now in demand.

One of the key issues in designing a triple-band GPS receiver is how to implement a highly integrated RF front-end that can operate at low power. To date, very few multi-band GPS receivers have been reported [4, 5, 6, 7, 8, 9]. Although dual-band receivers have been developed that use a band-selection architecture [6] or a simple parallel arrangement of several receivers [9], these approaches can not realize miniaturized low-power devices. Simultaneous reception with the same RF signal delay, which is important for some scientific applications, is also not possible [2].

In the present study, we propose an architecture for a triple-band GPS receiver that can simultaneously receive the L1, L2 and L5 frequency bands. The RF front-end for the proposed receiver is designed using $130 \mathrm{~nm}$ CMOS technology. The designed chip has a wide-band low-noise amplifier (LNA) with a low noise figure (NF) and input matching $\left(S_{11}\right)$ in the frequency range 1.15-1.6 GHz, and uses active and passive mixers for modified Weaver image rejection $[10,11]$.

\section{Triple-band receiver architecture}

The proposed receiver architecture is shown in Fig. 1 (a). It can separate signals in the three frequency bands passing through an RF signal path based on Weaver image rejection. It is a modified version of a previously proposed dual-band GPS receiver [10, 11]. By appropriate conversion of the phase of the signal in each of the three bands, the desired signal can be isolated at each port. By choosing appropriate frequencies for the local oscillators LO1 and LO2, simultaneous triple-band reception can be achieved. Two poly-phase filters (PPFs) are used to distinguish signals in the three bands concurrently. In the first PPF, signals in the L1 band are separated from the combined signals in the L2 and L5 bands using a Weaver image-rejection technique. Subsequently, the phases of the L2 and L5 signals are changed by the second PPF. This process allows the signals associated with each of the frequency bands to be separated to their own ports.

Details of the signal processing method used are shown in Fig. 1(b). It is 


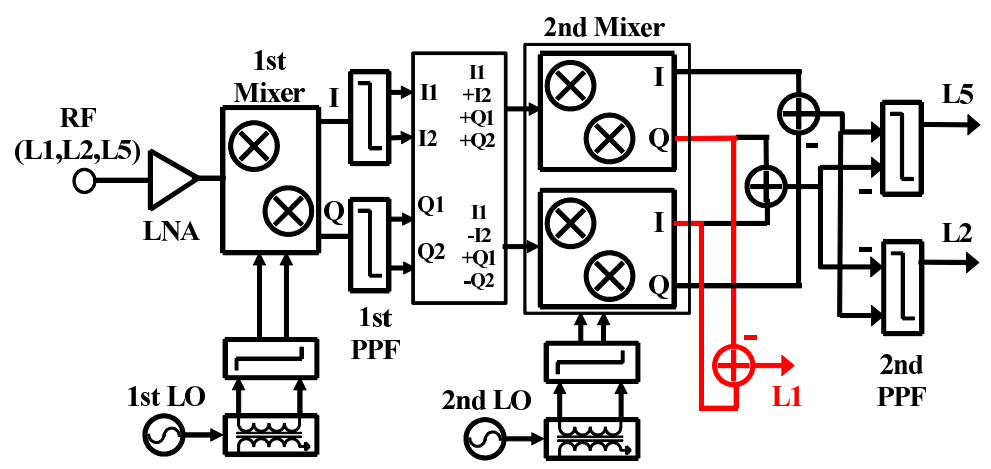

(a)

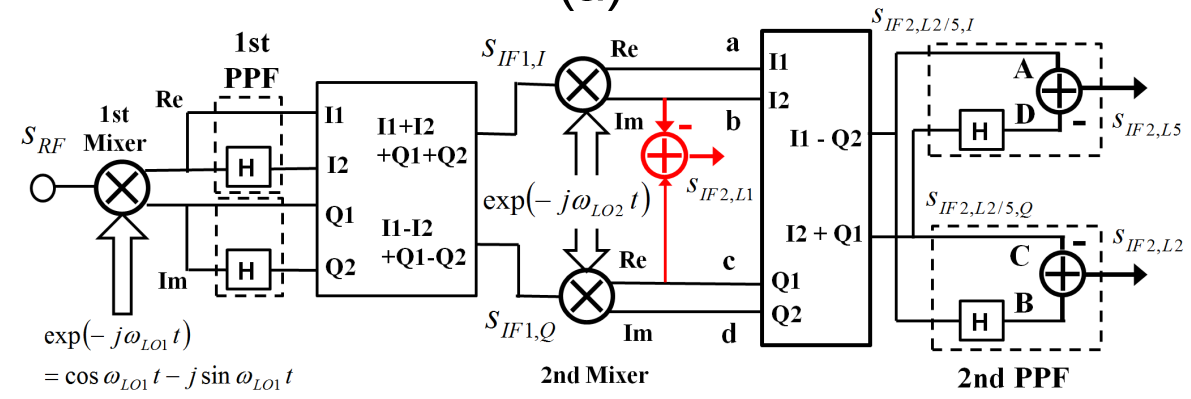

(b)
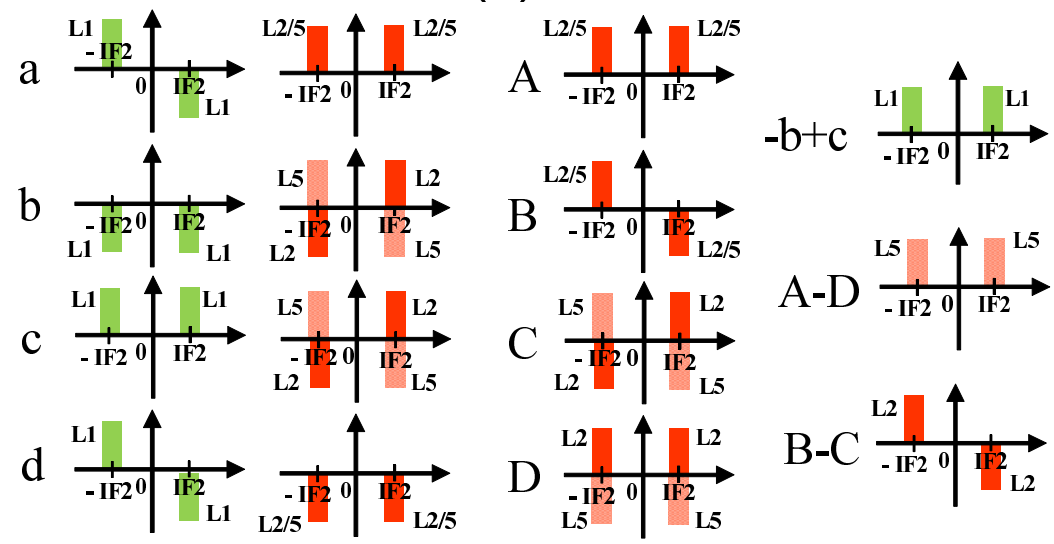

$\mathrm{D}$
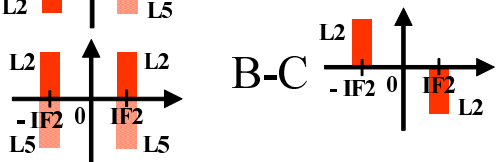

(c)

Fig. 1. (a) Triple-band receiver, (b) signal processing, and (c) conceptual signal spectrum at the locations indicated in (b).

based on the concept of complex signal processing and the Hilbert transform (denoted by "H" in the figure) [12, 13]. The RF input signal is expressed as $s_{R F}(t)=\Re[a(t) \exp (j \omega t)]$, where $\omega$ is the angular frequency and $a(t)$ is the complex baseband signal. In the first mixer, a quadrature LO signal with a frequency $f_{L O 1}\left(=\omega_{L O 1} / 2 \pi\right)$ convert $s_{R F}(t)$ to a complex signal given by

$$
s_{R F}(t) e^{-j \omega_{L O 1} t}=\frac{1}{2}\left[a(t) e^{j\left(\omega-\omega_{L O 1}\right) t}+a^{*}(t) e^{\left.-j\left(\omega+\omega_{L O 1}\right) t\right]} .\right.
$$

In the above equation, the desired down-converted component is represented by the first term. The first PPF performs a Hilbert transform [13] on the real and imaginary parts of the converted signals. The resistive adder then 
generates the following signals,

$$
\begin{aligned}
s_{I F 1, I}(t)= & \Re\left[s_{R F} e^{-j \omega_{L O 1} t}\right]+\Im\left[s_{R F} e^{-j \omega_{L O 1} t}\right] \\
& +H\left[\Re\left[s_{R F} e^{-j \omega_{L O 1} t}\right]\right]+H\left[\Im\left[s_{R F} e^{-j \omega_{L O 1} t}\right]\right], \\
s_{I F 1, Q}(t)= & \Re\left[s_{R F} e^{-j \omega_{L O 1} t}\right]+\Im\left[s_{R F} e^{-j \omega_{L O 1} t}\right] \\
& -H\left[\Re\left[s_{R F} e^{-j \omega_{L O 1} t}\right]\right]-H\left[\Im\left[s_{R F} e^{-j \omega_{L O 1} t}\right]\right] .
\end{aligned}
$$

Since the transfer function for the Hilbert transform is $-j \operatorname{sgn}(\omega)$, where $\operatorname{sgn}(\omega)$ represents the sign function, the above signals can be expressed as

$$
\begin{aligned}
s_{I F 1, I}(t)= & \Re\left[a(t) e^{j\left(\omega-\omega_{L O 1}\right) t}\right] u\left(\omega_{L O 1}-\omega\right) \\
& +\Im\left[a(t) e^{j\left(\omega-\omega_{L O 1}\right) t}\right] u\left(\omega-\omega_{L O 1}\right)+\Re\left[a(t) e^{j\left(\omega+\omega_{L O 1}\right) t}\right], \\
s_{I F 1, Q}(t)= & -\Im\left[a(t) e^{j\left(\omega-\omega_{L O 1}\right) t}\right] u\left(\omega_{L O 1}-\omega\right) \\
& +\Re\left[a(t) e^{j\left(\omega-\omega_{L O 1}\right) t}\right] u\left(\omega-\omega_{L O 1}\right)+\Im\left[a(t) e^{j\left(\omega+\omega_{L O 1}\right) t}\right],
\end{aligned}
$$

where $u(\omega)(=(1+\operatorname{sgn}(\omega)) / 2)$ is a step function. Note that the frequency components $\omega-\omega_{L O 1}$ in $s_{I F 1, I}(t)$ and $s_{I F 1, Q}(t)$ have the opposite polarity to each other and change their own polarity for $\omega>\omega_{L O 1}$ and $\omega<\omega_{L O 1}$. This provides a method for separation of the L1 and (L2 and L5) signals.

The second mixer, using a quadrature $\mathrm{LO}$ signal with a frequency $f_{\mathrm{LO} 2}(=$ $\left.\omega_{L O 2} / 2 \pi\right)$, causes a downward shift of the center frequency of the desired L1, L2, and L5 bands. Appropriate addition and subtraction of the outputs of the second mixer then extracts the L1 signal and produces a combined L2 and L5 signal with a different polarity, as follows:

$$
\begin{aligned}
s_{I F 2, L 1}(t)= & -\Im\left[s_{I F 1, I}(t) e^{-j \omega_{L O 2} t}\right]+\Re\left[s_{I F 1, Q}(t) e^{-j \omega_{L O 2} t}\right] \\
= & \Re\left[a(t) e^{j\left(\omega-\omega_{L O 1}-\omega_{L O 2}\right) t}\right] u\left(\omega-\omega_{L O 1}\right) \\
& -\Im\left[a(t) e^{j\left(\omega-\omega_{L O 1}-\omega_{L O 2}\right) t}\right] u\left(\omega_{L O 1}-\omega\right), \\
s_{I F 2, L 2 / 5, I}(t)= & \Re\left[s_{I F 1, I}(t) e^{-j \omega_{L O 2} t}\right]-\Im\left[s_{I F 1, Q}(t) e^{-j \omega_{L O 2} t}\right] \\
= & \Im\left[a(t) e^{j\left(\omega-\omega_{L O 1}+\omega_{L O 2}\right) t}\right] u\left(\omega-\omega_{L O 1}\right) \\
& +\Re\left[a(t) e^{j\left(\omega-\omega_{L O 1}+\omega_{L O 2}\right) t}\right] u\left(\omega_{L O 1}-\omega\right) \\
& +\Re\left[a(t) e^{j\left(\omega+\omega_{L O 1}-\omega_{L O 2}\right) t}\right], \\
s_{I F 2, L 2 / 5, Q}(t)= & \Im\left[s_{I F 1, I}(t) e^{-j \omega_{L O 2} t}\right]+\Re\left[s_{I F 1, Q}(t) e^{-j \omega_{L O 2} t}\right] \\
= & \Re\left[a(t) e^{j\left(\omega-\omega_{L O 1}+\omega_{L O 2}\right) t}\right] u\left(\omega-\omega_{L O 1}\right) \\
& -\Im\left[a(t) e^{j\left(\omega-\omega_{L O 1}+\omega_{L O 2}\right) t}\right] u\left(\omega_{L O 1}-\omega\right) \\
& +\Im\left[a(t) e^{j\left(\omega+\omega_{L O 1}-\omega_{L O 2}\right) t}\right] .
\end{aligned}
$$

Note that the frequency components $\omega-\omega_{L O 1}+\omega_{L O 2}$ in $s_{I F 2, L 2 / 5, I}(t)$ and $s_{I F 2, L 2 / 5, Q}(t)$ have the opposite polarity to each other. As described later, an appropriate combination of these frequency components and their Hilbert transforms can exhibit different polarities for $\omega>\omega_{L O 1}-\omega_{L O 2}$ and $\omega<$ $\omega_{L O 1}-\omega_{L O 2}$. This provides a method for separation of the L2 and L5 signals.

To convert the center frequencies of the L1, L2, and L5 bands to the same frequency $f_{I F 2}\left(=\omega_{I F 2} / 2 \pi\right)$ along the signal path from the first to the second 
mixer, the LO1 and LO2 frequencies $f_{\mathrm{LO} 1}, f_{\mathrm{LO} 2}$ are set as follows.

$$
\begin{aligned}
& \omega_{L O 1}=\left(\omega_{L 1}+\omega_{L 2}\right) / 2 \\
& \omega_{L O 2}=\omega_{L O 1}-\left(\omega_{L 2}+\omega_{L 5}\right) / 2,
\end{aligned}
$$

where $f_{L i}\left(=\omega_{L i} / 2 \pi\right)$ is the center frequency of the $L_{i}$ band $(i=1,2,5)$. In this case, $\omega_{I F 2}=\left(\omega_{L 2}-\omega_{L 5}\right) / 2$. Since $f_{L 1}=1575.42 \mathrm{MHz}, f_{L 2}=1227.6 \mathrm{MHz}$, and $f_{L 5}=1176.45 \mathrm{MHz}$, this gives $f_{L O 1}=1401.51 \mathrm{MHz}, f_{L O 2}=199.485 \mathrm{MHz}$, and $f_{I F 2}=25.575 \mathrm{MHz}$. In the first down-conversion and the first PPF, the L1 and L2 signals are converted to $\pm 173 \mathrm{MHz}$ images of each other with the opposite polarity, as indicated by the first and second terms in Eqs. (4) and (5). On the other hand, the L5 band signal is converted to $225.06 \mathrm{MHz}$ in this stage. With regard to the second down-conversion, Eq. (6) indicates that $s_{I F 2, L 1}(t)$ at $f_{I F 2}$ contains the L1 signal. Similarly, as seen in Eqs. (7) and (8), the second terms in $s_{I F 2, L 2 / 5, I}(t)$ and $s_{I F 2, L 2 / 5, Q}(t)$ at $\pm f_{I F 2}$ contain the L2 and L5 signals. These signals are shown graphically in Fig. 1 (c).

To separate the L2 and L5 signals after the second mixer, $s_{I F 2, L 2 / 5, I}(t)$, $s_{I F 2, L 2 / 5, Q}(t)$ and their Hilbert transforms generated in the second PPF, which are shown in Fig. 1 (c), are manipulated as follows,

$$
\begin{aligned}
s_{I F 2, L 2}(t)= & -s_{I F 2, L 2 / 5, Q}(t)+H\left[s_{I F 2, L 2 / 5, I}(t)\right] \\
= & 2 \Im\left[a(t) e^{j\left(\omega-\omega_{L O 1}+\omega_{L O 2}\right) t}\right] u\left(\omega_{L O 1}-\omega\right) u\left(\omega-\omega_{L O 1}+\omega_{L O 2}\right) \\
& -2 \Re\left[a(t) e^{j\left(\omega-\omega_{L O 1}+\omega_{L O 2}\right) t}\right] u\left(\omega-\omega_{L O 1}\right) \\
s_{I F 2, L 5}(t)= & s_{I F 2, L 2 / 5, I}(t)-H\left[s_{I F 2, L 2 / 5, Q}(t)\right] \\
= & 2 \Re\left[a(t) e^{j\left(\omega-\omega_{L O 1}+\omega_{L O 2}\right) t}\right] u\left(\omega_{L O 1}-\omega_{L O 2}-\omega\right) \\
& +2 \Re\left[a(t) e^{j\left(\omega+\omega_{L O 1}-\omega_{L O 2}\right) t}\right]
\end{aligned}
$$

where the fact that $\omega_{L O 1}>\omega_{L O 2}$ is used to simplify expressions such as $u\left(\omega-\omega_{L O 1}\right) u\left(\omega-\omega_{L O 1}+\omega_{L O 2}\right)=u\left(\omega-\omega_{L O 1}\right)$. Eqs. (11) and (12) reveal that $s_{I F 2, L 2}(t)$ and $s_{I F 2, L 5}(t)$ at $f_{I F 2}$ contain the L2 and L5 signals, respectively, as seen in Fig. 1 (c). The last term in $s_{I F 2, L 1}(t)$ (Eq. (6)), $s_{I F 2, L 2}(t)$ (Eq. (11)), and $s_{I F 2, L 5}(t)$ (Eq. (12)), corresponding to higher frequency components, can be filtered out in the following stage (not shown in Fig. 1).

\section{Circuit design}

For the proposed GPS receiver that is capable of operating over a wide frequency range of $1.17-1.6 \mathrm{GHz}$, the LNA is one of the most important building blocks. In this study, a single-ended LNA with an active balun was used to obtain a differential output signal for the first mixer, as shown in Fig. 2 (a). Use of this topology can reduce the phase and amplitude errors of the differential output signals. The LNA is based on a cascode common-source amplifier with an inductive source degeneration topology [5]. The presence of only a single gain stage reduces the power required and maintains high linearity, and the cascode transistor isolates the input and output ports. The values of the external input inductance $L_{g}$ and the on-chip inductance $L_{s}$ are chosen to achieve stable input matching. In contrast to previous work [5], 


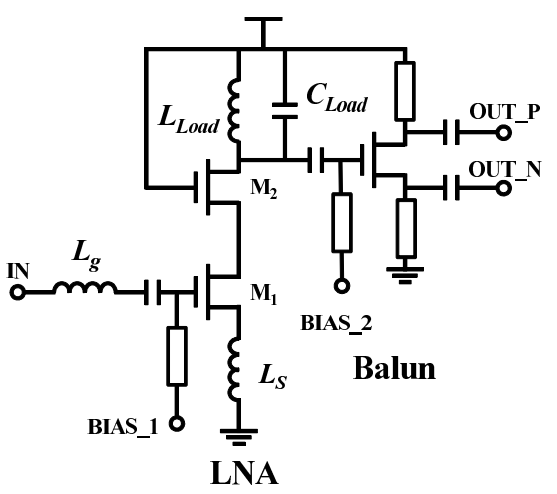

(a)

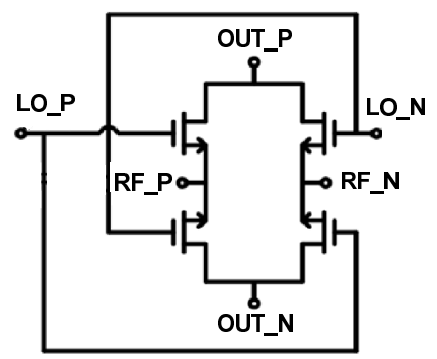

(c)

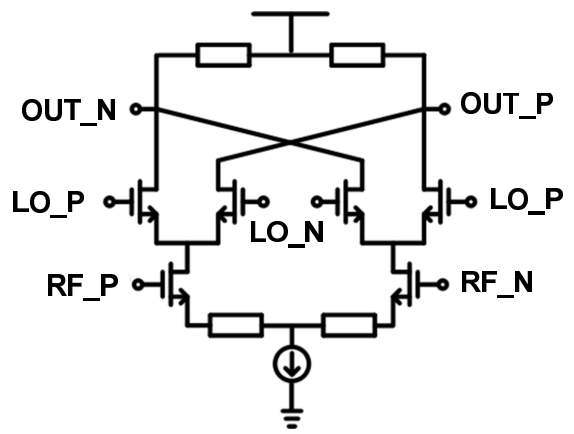

(b)

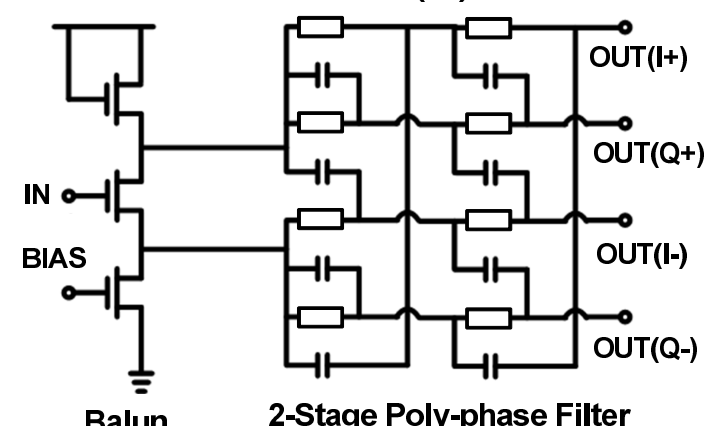

(d)

Fig. 2. Circuit schematic of (a) LNA, (b) first mixer, (c) second mixer, and (d) quadrature LO generator designed in this work.

the quality factor of the load tank circuit is reduced only by the parasitic resistance of the on-chip inductance $L_{\text {Load }}$.

The NF and input reflection coefficient $\left|S_{11}\right|$ determine the overall receiver characteristics. The following expressions for NF and $\left|S_{11}\right|$ are used,

$$
\begin{aligned}
N F & \simeq 1+\frac{\gamma}{\alpha} \cdot \chi \cdot R_{s} \cdot g_{m} \cdot\left(\frac{\omega_{0}}{\omega_{T}}\right)^{2}, \\
\chi & =1-2|c| \alpha \sqrt{\frac{\delta}{\kappa \gamma}}+\frac{\delta \alpha^{2}}{\kappa \gamma}\left(1+Q_{i n}^{2}\right), \\
\left|S_{11}\right| & =\left|\frac{j Q_{i n}\left|\frac{\omega}{\omega_{0}}-\frac{\omega_{0}}{\omega}\right|}{2+j Q_{i n}\left|\frac{\omega}{\omega_{0}}-\frac{\omega_{0}}{\omega}\right|}\right|
\end{aligned}
$$

where $R_{s}$ is the signal source impedance, $f_{0}\left(=\omega_{0} / 2 \pi\right)$ is the operating frequency, $f_{T}\left(=\omega_{T} / 2 \pi\right)$ is the unity gain frequency of device $\mathrm{M}_{1}, Q_{i n}$ is a function of the gate width of M1, which should be chosen to achieve a sufficiently small input reflection [5], $\alpha$ is the ratio of the device transconductance to the zero-bias drain conductance, $\kappa$ is the Elmore constant, and $\gamma, \delta$, and $c$ are the drain and induced gate noise current factors and their correlation coefficient, respectively $[14,15]$. Based on these conditions, $Q_{i n}$ can be optimized to achieve a low NF in all three frequency bands for a given current consumption ( $3 \mathrm{~mA}$ in this work). Simulations carried out using Cadence Spectre revealed that $\left|S_{11}\right|<-10 \mathrm{~dB}$ and NF $<1.5 \mathrm{~dB}$ for the L1, L2, and 
L5 bands (1150 $1600 \mathrm{MHz})$, as shown in Fig. 3 (c).

Figures $2(\mathrm{~b})$ and (c) show circuit schematics for the first and second mixers. To improve the linearity for use in a wide-band GPS system, the transconductance stage in the first mixer has source degeneration. In contrast to our previous work $[10,11]$, a shared transconductor is not used in the first mixer because the image rejection ratio (IMRR) can also be improved in the IF circuit blocks [16]. The quadrature LO generator shown in Fig. 2 (d) is used for the first and second mixing processes.

\section{Experimental results}

An optical micrograph of the chip fabricated using a $130 \mathrm{~nm}$ CMOS process is shown in Fig. 3 (a). The chip area including input and output pads is $2.4 \mathrm{~mm}^{2}$. The power consumption is about $7.2 \mathrm{~mW}$ with a $1.2 \mathrm{~V}$ supply voltage, which is mainly for the LNA and mixers.

Measurements were carried out using a wafer probe station. The designed chip had no external inductor $\left(L_{g}\right.$ in Fig. $2(\mathrm{a})$ ) dominating input matching. To avoid instrumental errors originating from bonding wires, the small-signal and noise characteristics of the receiver chip with an ideal inductor $L_{g}$ in series with the gate of $\mathrm{M}_{1}$ were calculated on the basis of measured data [17]. The insertion loss for the input matching circuit, cable and probe needle was also compensated for [18]. The resulting $S_{11}$ and NF data are shown in Figs. 3 (b) and (c). The NF for the receiver chip is less than $7.1 \mathrm{~dB}$ over a wide frequency range covering the L1, L2, and L5 bands.

To investigate the capability of the chip for triple-band reception, the frequency selectivity was measured, and the results are shown in Fig. 3 (d). The input power was $-50 \mathrm{dBm}$ and the power of the LO1 and LO2 signals was $5 \mathrm{dBm}$. The output signal power level was found to be about $-50 \mathrm{dBm}$. The IMRR for the L1 and (L2, L5) signals was about 40-42 dB and that for the L2 and L5 signals was about 35-37 dB.

\section{Conclusion}

In this letter, a receiver is proposed for concurrent triple-band reception of L1, L2 and L5 GPS signals using two local oscillators. The proposed receiver is designed using a $130 \mathrm{~nm}$ CMOS process to demonstrate the possibility of concurrent triple-band reception using a modified Weaver image-rejection technique. For each of the output ports, the IMRR was estimated to be $35-42 \mathrm{~dB}$.

Although the present study is preliminary and further work is required before the device can be used in practical applications, the feasibility of simultaneous GPS triple-band reception with a single RF path has been demonstrated.

\section{Acknowledgments}

This chip design is supported by the VLSI Design and Education Center (VDEC), the University of Tokyo, in collaboration with Agilent Technologies 


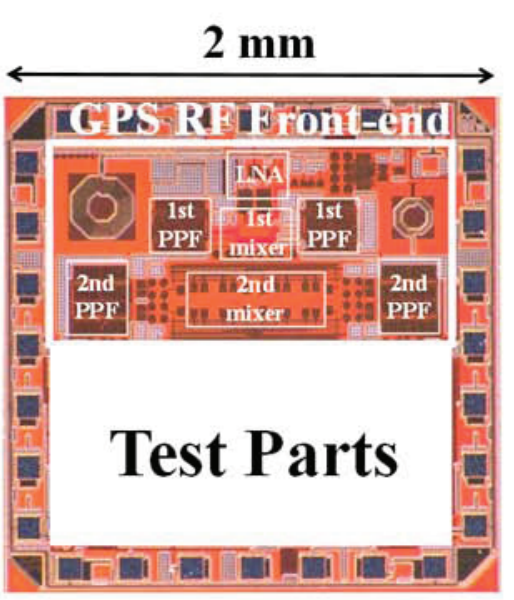

(a)

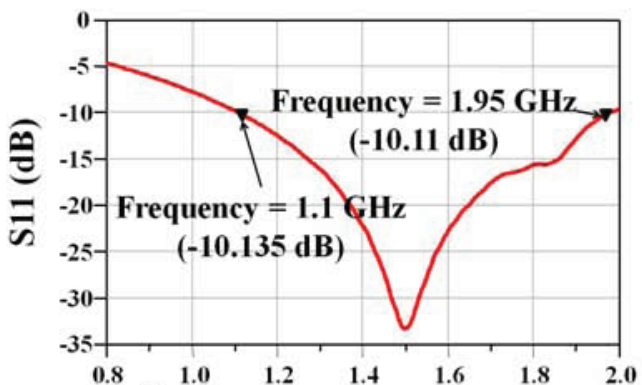

(b) Frequency $(\mathbf{G H z})$

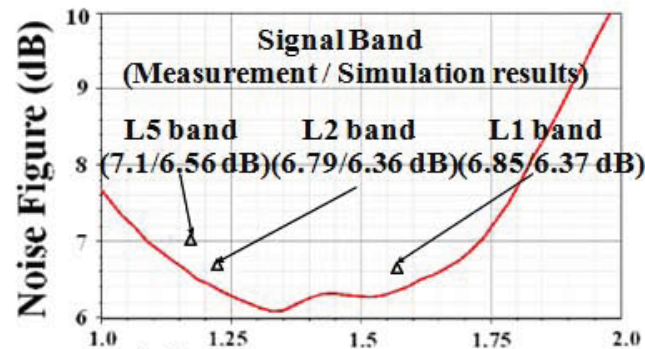

(c) Frequency $(\mathbf{G H z})$

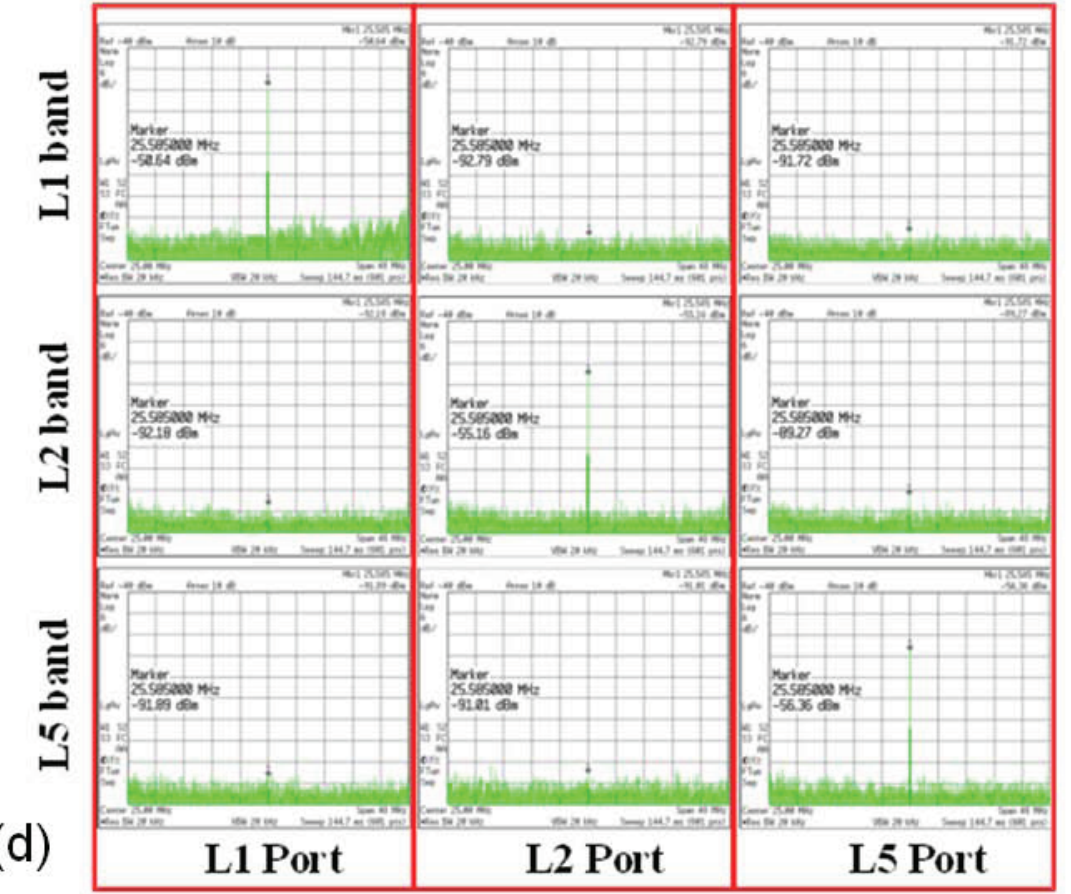

Fig. 3. (a) Optical micrograph of fabricated chip, (b) measured $S_{11}$, (c) measured NF (with simulated result), (d) measured output spectrum at each port for an unmodulated input signal in each of the frequency bands.

Japan, Ltd. and Cadence Design Systems, Inc. This work is also supported in part by the Japan Society for the Promotion of Science (JSPS) for Grantsin-Aid for Young Scientists $(A)(\sharp 22686083)$. 